\title{
A case of distal limb arterial tortuosity and dilation: observations and potential clinical significance
}

\author{
Y. Carter ${ }^{1}$, D.J. Bennett ${ }^{2}$, V. Molla ${ }^{2}$, A.E. Wink ${ }^{1}$, A.J. Collins ${ }^{1}$, E.L. Giannaris ${ }^{1}$ \\ 'Division of Translational Anatomy, Department of Radiology, University of Massachusetts Medical School, \\ Worcester, MA, United States of America \\ 2Undergraduate Medical Education, University of Massachusetts Medical School, Worcester, MA, \\ United States of America
}

[Received: 29 January 2021; Accepted: 22 April 2021; Early publication date: 25 May 2021]

\begin{abstract}
Arterial tortuosity describes variation via bending of the arterial wall and has been noted in several arteries throughout the body. Tortuous blood vessels can cause nerve compression, as well as present difficulties to surgeons and radiologists. Here we present an unusual case of multi-vessel arterial tortuosity discovered in 78-year-old Hispanic male cadaver, independent of systemic pathology. The left ulnar and right tibial arteries were dissected, and using calibrated digital callipers, their external and internal diameters were measured both at the origin site and at the site of greatest dilation. Both wall thickness and the number of inflection points were also measured. Six bends were noticed in the ulnar artery and its diameter measured $8.11 \mathrm{~mm}$ at its widest, with a wall thickness of $0.88 \mathrm{~mm}$. On the lower extremity, the right tibial artery had three bends and its diameter measured $4.86 \mathrm{~mm}$ at its widest, with a wall thickness of $1.32 \mathrm{~mm}$. This uncommon tortuosity is not only more prone to laceration during surgery, but the bending and thickening can be mistaken for tumours. Finally, fluid dynamics can be altered, resulting in an impact on blood pressure in the extremities. Thus, raising awareness is crucial to prevent both symptoms and iatrogenic complications. (Folia Morphol 2022; 81, 3: 791-797)
\end{abstract}

Key words: arterial, tortuosity, ulnar, tibial, cadaveric

\section{INTRODUCTION}

Arterial tortuosity involves varying degrees of abnormal twists and bends [7]. There are a few arteries known for their tortuous course, including the classic descriptions of the splenic and facial arteries [40]; however, there have been numerous reports of incidental findings of anatomical variation in arteries throughout all regions of the body including the head and neck [6, 14], thorax [42], abdomen and pelvis [27], upper limb and lower limb [45, 46]. These incidental cases report findings generally with involvement of a single vessel are distinct from arterial tortuosity syndrome, which is characterized by "severe and widespread arterial tortuosity of the aorta and middle-sized arteries" plus craniofacial involvement and skin or connective tissue disorder [5]. While the exact causal mechanisms of arterial tortuosity are unknown, it has been associated with senescence, high blood pressure as well as other cardiovascular risks, and is more common in females [7].

Address for correspondence: Y. Carter, PhD, Department of Radiology, University of Massachusetts Medical School, 55 Lake Avenue North Worcester MA 01655, United States of America, tel: 508450 3412, e-mail: yasmin.carter@umassmed.edu

This article is available in open access under Creative Common Attribution-Non-Commercial-No Derivatives 4.0 International (CC BY-NC-ND 4.0) license, allowing to download articles and share them with others as long as they credit the authors and the publisher, but without permission to change them in any way or use them commercially. 
Clinically, both arterial and venous abnormal tortuosity has been associated with compression of nerves $[11,15,26,49]$. A tortuous ulnar artery may also present difficulties to neuroradiologists, radiologists and surgeons during relevant procedures $[36,44]$. Regarding the lower limb, previous reports had no comment on clinical implications of posterior tibial arterial tortuosity; though tortuosity of the accompanying posterior tibial veins has been linked to entrapment of the tibial nerve within the tarsal tunnel [31].

Here we report and discuss a unique case of distal limb arterial tortuosity discovered during a routine cadaveric dissection. While there have been reports in distal limb vessels, to our knowledge, these have not been reported in the same individual outside of arterial tortuosity syndrome.

\section{CASE REPORT}

The presence of an enlarged and tortuous ulnar artery was observed during a routine dissection of a left upper limb in a 78-year-old Hispanic male cadaver. The left ulnar artery had a typical appearance at its origin from the brachial artery; however, $\sim 66 \mathrm{~mm}$ proximal to the flexor retinaculum, a significant winding pattern was observed with sinusoidal bends and a widened appearance (Fig. 1). Further dissection revealed a mildly enlarged but not tortuous artery on the right side (Fig. 2). Dissection of the lower extremities revealed a similar pattern in the tibial artery with enlargement and tortuosity noted on the right side (Fig. 3), while the left appeared relatively normal. Further inspection and dissection of the arteries in all other regions were unremarkable.

In a questionnaire filled out prior to the donor's death, they self-reported a brief smoking history over 50 years prior during young adulthood as well as a cancer history but did not specify which type. No enlarged lymph nodes or abnormal masses were seen. The University of Massachusetts Medical School (UMMS) Institutional Review Board determined this case report (\#H00020805) is not human subject research.

\section{METHODS}

Terminology for describing vessel appearance and classification of the anatomical variation is based on Ciurica et al. [7]. While there is currently no standard-

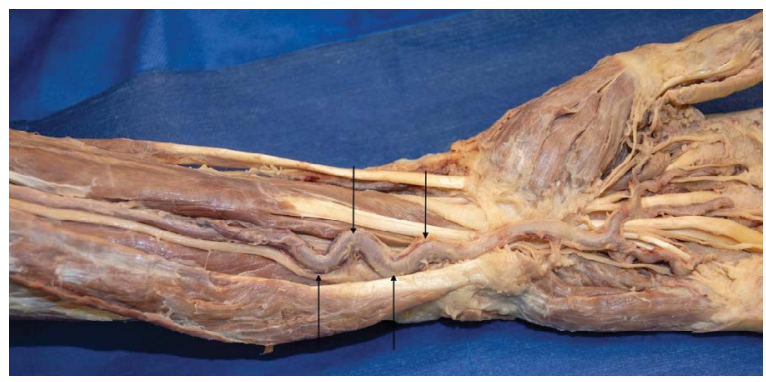

Figure 1. Ulnar artery on left side. Arrows signify abnormal sinusoidal bends.

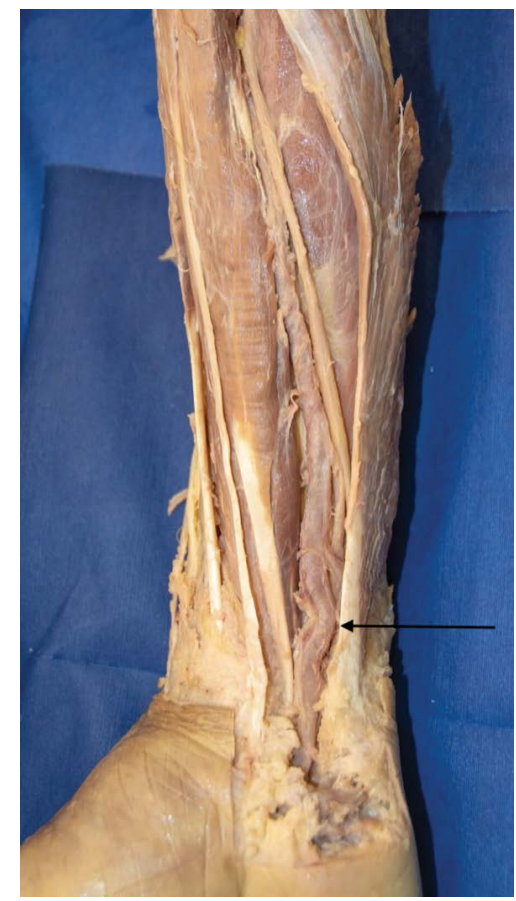

Figure 2. Ulnar artery on right side. There was mild external enlargement and a slight winding nature.

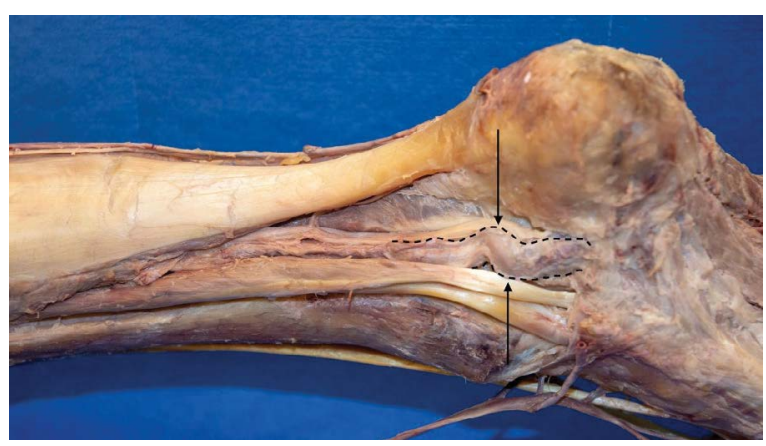

Figure 3. Tibial artery on right side. Arrows signify abnormal sinusoidal bends. Dotted lines highlight the area of external enlargement and a slight winding nature. 
Table 1. Results of measurements of arteries in millimetres

\begin{tabular}{lccc}
\hline Measure & Left ulnar artery & Right ulnar artery & Right tibial artery \\
\hline SLM & $132.06 \pm 2.14$ & $75.45 \pm 3.14$ & $115.73 \pm 1.27$ \\
Path & $151.86 \pm 3.94$ & $93.98 \pm 0.91$ & $130.22 \pm 1.97$ \\
Number of inflections & $6 \pm 0$ & $3 \pm 0$ & $4 \pm 0$ \\
External MD origin & $4.66 \pm 0.11$ & $5.70 \pm 0.26$ & $3.47 \pm 0.04$ \\
External MD - widest point & $8.11 \pm 0.49$ & $8.16 \pm 0.42$ & $4.86 \pm 0.11$ \\
Internal MD - origin & $4.58 \pm 0.06$ & - & $3.66 \pm 0.11$ \\
Internal MD - widest point & $4.07 \pm 0.11$ & - & $2.77 \pm 0.07$ \\
Wall thickness - origin & $0.57 \pm 0.05$ & - & $0.81 \pm 0.01$ \\
Wall thickness - widest point & $0.88 \pm 0.01$ & - & $1.32 \pm 0.03$ \\
\hline
\end{tabular}

Data are shown as average \pm standard deviation; $M D$ — maximal diameter; SLM — straight-line measure

ised measure for determining the amount of arterial tortuosity in two-dimensions, three main methods are used consistently in the literature. The first method is based on a sum-of-angles metric, typically defined as the deviation from the anatomical normal straight path of the vessel. This metric is usually provided as a total number of degrees or as a ratio of straight-line length $[12,35,48]$. The second method calculates the degree of angulation from a centre line of flow, often as a mathematical measure of curvature, or amplitude [4]. This measure can be thought of as representing 'wave-height'. Lastly is the 'distance metric' which provides a ratio of the vessel path to the straight-line measure and can be used to describe the actual length of the vessel versus the anatomical normal length. In cadaveric studies this usually involves excision and straightening of the vessel before measuring $[10,41]$. Distance metric was chosen as the optimal assessment of vessel tortuosity for this study as it would more accurately represent the normally straight arteries involved. To achieve this, measures were collected for straight-line length and path. The two vessels with greatest variation (left ulnar artery and right tibial artery) were subsequently removed for further analysis. For these, vessel external and internal (lumen) diameter was measured at both the origin and at the site of greatest dilation, wall thickness was also determined at the same sites. Additionally, the number of inflection points (bends) was noted for all vessels. All of the measures were taken by two observers with calibrated digital callipers.

\section{RESULTS}

The diameter of the tortuous segment of the ulnar artery measured $8.11 \mathrm{~mm}$ at its widest, whereas it was only $4.66 \mathrm{~mm}$ at its origin (Table 1 ). The arterial walls of the tortuous segment appeared thickened, and the lumen of the artery measured $4.2 \mathrm{~mm}$ (Fig. 2). Six bends were noted in the left ulnar artery prior to diving under the palmar carpal ligament and continuing into the ulnar tunnel (Guyon's canal). Upon continued dissection of the hand, the ulnar artery terminated into the superficial palmar arch and common palmar digital arteries. The tortuosity seen in the ulnar artery continued into the distal palmar arteries. In the area of tortuosity, the wall thickness was greater $(0.88 \mathrm{~mm})$ than at the origin (0.47), resulting in a reduced internal diameter of the lumen $(4.07 \mathrm{~mm}$ vs. $4.58 \mathrm{~mm}$ ). The left ulnar artery was then traced proximally and was noted to be typical in size and shape proximal to the bends, with no aneurysms apparent. No abnormalities were seen at the origin of the vessel from the brachial artery. The brachial artery was also traced proximally and had a typical appearance. Dissection of the right arm revealed a slightly enlarged ulnar artery which had a diameter of $5.70 \mathrm{~mm}$ at the origin (Fig. 2). The right ulnar artery also exhibited a slight bend, $30 \mathrm{~mm}$ proximal of Guyon's canal, but a torturous pattern did not exist.

Dissection of the lower extremities revealed a tortuous tibial artery on the right side (Fig. 3) with the left appearing normal. The diameter of the tortuous segment of the right tibial artery measured $4.86 \mathrm{~mm}$ at its widest and $3.47 \mathrm{~mm}$ at its origin. Again, in the area of tortuosity, the wall thickness was much larger $(1.32 \mathrm{~mm})$ than at the origin $(0.81)$, resulting in a reduced internal diameter of the lumen $(2.77 \mathrm{~mm}$ vs. $3.66 \mathrm{~mm}$ ). No other vascular variants were observed. 


\section{DISCUSSION}

Numerous distal limb arterial variations have been reported in the literature [17, 21, 32, 38, 44, 49]. The present case reports a cadaveric dissection revealing distally tortuous ulnar and posterior tibial arteries, without any other vascular abnormalities. This is a particular import given that multi-site tortuosity is associated with systemic pathologies such as arterial tortuosity syndrome. While the exact cause of arterial tortuosity is unknown, several mechanisms have been suggested including calcification of the arterial wall as well as inconsistent arterial repair mechanisms [13]. Additionally, it can be related to age, loss of arterial elasticity, and position relative to the joint axis [45]. Both genetics and vascular pathologies, including hypertension and atherosclerosis, may play a role [13]. The presence of tortuous tibial arteries as well could suggest a systemic pathology, or genetic cause, as the reason for the tortuosity in this donor.

The ulnar artery is one of two terminal branches of the brachial artery. It arises from the brachial artery in the medial aspect of the forearm, typically distal to the antecubital fossa, and is the primary source of blood supply of the medial forearm. Typically, it is accompanied by the median nerve in the proximal forearm and by the ulnar nerve medially in the distal forearm and wrist. The ulnar artery terminates at the superficial palmar arch, where the arch anastomoses with a branch of the radial artery, the other terminal branch of the brachial artery [39]. The posterior tibial artery is the more posterior branch of the popliteal artery and supplies the posterior compartment of the leg. It gives rise to the fibular artery and terminally bifurcates into the medial and lateral plantar arteries. The posterior tibial artery courses with the tibial nerve and accompanying veins in the tarsal tunnel.

Variations in the upper limb vasculature have been well documented in the literature. Previous studies have described and classified upper limb arterial variations such as a superficial brachial artery, an accessory brachial artery, a superficial radial artery, and arteries with higher origins from the brachial artery, among others [22, 30, 38]. For the ulnar artery, thrombosis and aneurysmal dilatations are the most common [2]; other variations include proximal origins and superficial courses. There have been well-documented cases of variations such as a superficial ulnar artery, which poses a significant issue during forearm free flap harvesting $[2,3,28]$ yet there are only a limited number of cases reporting a tortuous ulnar artery. It has been reported that a looped and tortuous ulnar artery is the rarest anatomical variation seen in the ulnar artery [44].

Arterial tortuosity has also been described in the lower limb. Tortuosity has also been reported in the popliteal artery and tibial-peroneal trunk [34]. Reported variation of the posterior tibial artery is primarily related to the level of its origin or terminal bifurcation, as well as aplasia or hypoplasia [20]. Studies examining tortuosity in the posterior tibial artery reported a prevalence of $26-35 \%[16,18]$; these studies focused on the region surrounding the tarsal tunnel.

Using ultrasound and Doppler, Ashraf et al. [1] determined that the mean average internal diameter of the ulnar and radial arteries were $2.4 \pm 0.4 \mathrm{~mm}$ and $2.3 \pm 0.4 \mathrm{~mm}$ on the right and $2.3 \pm 0.3 \mathrm{~mm}$ and $2.2 \pm 0.4 \mathrm{~mm}$ on the left. A cadaveric study also reports the mean internal diameter of the ulnar and radial arteries to be 2.5 and $3.2 \mathrm{~mm}$ on the right and 2.4 and $3.0 \mathrm{~mm}$ on the left [37]. We report an internal diameter of $4.2 \mathrm{~mm}$ of the left ulnar artery with an $8 \mathrm{~mm}$ external diameter suggesting that the tortuosity increases artery lumen and overall size. Early computational fluid dynamics studies in tortuous coronary arteries suggest that such changes may impede the ability of the artery to self-regulate blood flow during increased demand [47]. Nevertheless, tortuous distal limb arteries carry significant clinical implications.

\section{Clinical implications}

Vascular lesions in the ulnar artery, including a tortuous artery, can result in nerve compression. Reports have linked a tortuous ulnar artery to Guyon's canal syndrome, a condition that causes hand numbness, pain, and tingling, resulting in a significant decline in quality of life [9]. The canal of Guyon, also called the distal ulnar tunnel, is a fibro-osseous space containing the ulnar nerve and its branches, the ulnar artery, as well as veins and lymphatic vessels [8]. Symptoms may be caused by ulnar nerve entrapment in Guyon's canal, due to the proximity of the nerve to the malshaped ulnar artery $[11,15,49]$. This is also true of the tarsal tunnel in the lower extremity. Machiels et al. [25] reported that dilated or tortuous veins in the tarsal tunnel can cause compression of the posterior tibial nerve resulting in tarsal tunnel syndrome. The variation seen here in the posterior tibial artery may also have implications for tibial nerve blocks and calcaneal pin insertions. A tortuous posterior tibial 
artery is exceedingly rare in the literature, but it is reasonable to conclude that such an artery can compress the posterior tibial nerve and cause symptoms.

Tortuous vessels are problematic during surgery, imaging, and interventional procedures. On imaging, tortuous arteries can appear as solid masses, which can be mistaken for tumours [26]. A winding and tortuous artery is also more prone to laceration during surgery [26]. Further, the multiple bends seen in these vessels are at risk of perforation in various procedures [44]. The ulnar artery is also becoming more frequently used for percutaneous coronary intervention if the radial artery is deemed unusable [43]. In this circumstance, understanding the exact shape of the ulnar artery is necessary. In addition, the ulnar artery can be used for cerebral angiography under certain circumstances [23]. Finally, in patients undergoing dialysis, the ulnar artery can be used for creating arterio-venous fistulas [24].

In the aforementioned cases, knowledge of the ulnar artery's actual shape and positioning is crucial. Ultrasound and magnetic resonance imaging can be used to determine this information when necessary, in attempts to reduce both the symptoms of disorders as well as iatrogenic mishaps.

The abnormal variation in the arterial pattern noted here would have an impact on blood pressure to the extremities. Blood is an incompressible viscous fluid, and basic fluid flow mechanics principles can be used to extrapolate the impact of the variation on velocity and pressure [29]. The Hagen-Poiseuille equation is used to calculate the flow of viscous fluid in a cylindrical pipe [29].

As per Poiseuille's law, the viscosity of blood will cause a pressure drop, proportional to the length travelled [29], therefore the longer path created by the tortuosity seen here would result in lowered blood pressure travelling through the artery. While the vessels appear externally to be enlarged at the site of tortuosity this is an impact of increased wall thickness which actually results in a decrease in diameter of the lumen. When blood flows out of a larger circular pipe, into a narrower diameter its velocity will be increased; however, its pressure will decrease [33]. The narrowing of the vessel and the elongated path of the tortuosity could then be described cumulatively acting to result in decreased pressure. Additionally a decrease in pressure would be created by the inflection points (bends) [19].

\section{Limitations of the study}

The analyses presented here had a number of limitations, importantly the vessel's external and internal diameter was measured in two dimensions, essentially a representation of flattened width versus true diameter but this was internally controlled as all were measured in the same manner. Additionally, the dryness of vessels as part of the embalming and dissection process may have led to some shrinkage of the tissues. While vessels from both sides of the body were able to be measured for the upper limb it was not possible to take measurements from the contralateral lower limb as the donor was cremated and returned due to the family's urgent request.

\section{CONCLUSIONS}

The current report suggests that arterial tortuosity can occur in multiple sites distinct from the systemic, arterial tortuosity syndrome. A proper understanding of a patient's specific regional anatomy is vital for radiologists, surgeons, and clinicians in general, as unknown variations can lead to unexplained pain and iatrogenic injuries. Raising awareness regarding ulnar artery tortuosity should help to reduce such events.

\section{Acknowledgements}

The authors sincerely thank those who donated their bodies to science so that anatomical research could be performed. Results from such research can potentially increase humanity's overall knowledge that can then improve patient care. Therefore, these donors and their families deserve our highest gratitude.

Conflict of interest: None declared

\section{REFERENCES}

1. Ashraf T, Panhwar Z, Habib S, et al. Size of radial and ulnar artery in local population. J Pak Med Assoc. 2010; 60(10): 817-819, indexed in Pubmed: 21381609.

2. Bell RA, Schneider DS, Wax MK. Superficial ulnar artery: a contraindication to radial forearm free tissue transfer. Laryngoscope. 2011; 121(5): 933-936, doi: 10.1002/ lary.21465, indexed in Pubmed: 21520105.

3. Bondaz M, Lepivert JC, Majoufre-Lefebvre C, et al. À propos d'un cas d'artère ulnaire superficielle. Rev Stomatol Chir Maxillofac Chir Orale. 2016; 117(3): 173-175, doi: 10.1016/j.revsto.2016.04.001.

4. Bullitt E, Gerig G, Pizer SM, et al. Measuring tortuosity of the intracerebral vasculature from MRA images. IEEE Trans 
Med Imaging. 2003; 22(9): 1163-1171, doi: 10.1109/ TMI.2003.816964, indexed in Pubmed: 12956271.

5. Callewaert B, De Paepe A, Coucke P. Arterial Tortuosity Syndrome. University of Washington, Seattle; 1993. http:// www.ncbi.nlm.nih.gov/pubmed/25392904 (Accessed October 16, 2020).

6. Choi G, Han SH, Choi JO. Tortuous common carotid artery encountered during neck dissection. Eur Arch Otorhinolaryngol. 1998; 255(5): 269-270, doi: 10.1007/ s004050050056, indexed in Pubmed: 9638471.

7. Ciurică S, Lopez-Sublet M, Loeys BL, et al. Arterial Tortuosity. Hypertension. 2019; 73(5): 951-960, doi: 10.1161/ HYPERTENSIONAHA.118.11647, indexed in Pubmed: 30852920.

8. Depukat P, Mizia E, Klosinski M, et al. Anatomy of Guyon's canal: a systematic review. Folia Med Cracov. 2014; 54(2): 81-86, indexed in Pubmed: 25648313.

9. Depukat P, Mizia E, Kuniewicz M, et al. Syndrome of canal of Guyon: definition, diagnosis, treatment and complication. Folia Med Cracov. 2015; 55(1): 17-23, indexed in Pubmed: 26774628.

10. Dowson N, Boult M, Cowled P, et al. Development of an automated measure of iliac artery tortuosity that successfully predicts early graft-related complications associated with endovascular aneurysm repair. Eur J Vasc Endovasc Surg. 2014; 48(2): 153-160, doi: 10.1016/j.ejvs.2014.04.033, indexed in Pubmed: 24939664.

11. Emel E, Guzey KF, Alatas I. Guyon's canal syndrome due to tortuous ulnar artery: a case report. Turk Neurosurg. 2003; 13: 107-110.

12. Faggioli G, Ferri M, Gargiulo $M$, et al. Measurement and impact of proximal and distal tortuosity in carotid stenting procedures. J Vasc Surg. 2007; 46(6): 1119-1124, doi: 10.1016/j.jvs.2007.08.027, indexed in Pubmed: 18154988.

13. Han HC. Twisted blood vessels: symptoms, etiology and biomechanical mechanisms. J Vasc Res. 2012; 49(3): 185-197, doi: 10.1159/000335123, indexed in Pubmed: 22433458.

14. Iwanaga J, Watanabe K, Tsuyoshi S, et al. Tortuous common carotid artery: a report of four cases observed in cadaveric dissections. Case Rep Otolaryngol. 2016; 2016: 2028402, doi: 10.1155/2016/2028402, indexed in Pubmed: 27818819.

15. Jose RM, Bragg T, Srivastava S. Ulnar nerve compression in Guyon's canal in the presence of a tortuous ulnar artery. J Hand Surg Br. 2006; 31(2): 200-202, doi: 10.1016/j. jhsb.2005.10.003, indexed in Pubmed: 16290914.

16. Joshi SS, Joshu SD, Athavale S. Anatomy of tarsal tunnel and its applied significance. J Anat Soc India. 2006; 55(1): 52-56.

17. Kalisman M, Laborde K, Wolff TW. Ulnar nerve compression secondary to ulnar artery false aneurysm at the Guyon's canal. J Hand Surg Am. 1982; 7(2): 137-139, doi: 10.1016/s0363-5023(82)80077-4, indexed in Pubmed: 7069168

18. Kalpana R. Neurovascular branching pattern in the tarsal tunnel with reference to medio malleolar calcaneal axis: a cadaveric study. PhD diss. Published online. 2014.

19. Keulegan GH, Hilding Beij K. Pressure losses for fluid flow in curved pipes. US Government Printing Office. 1937.
20. Kil SW, Jung GS. Anatomical variations of the popliteal artery and its tibial branches: analysis in 1242 extremities. Cardiovasc Intervent Radiol. 2009; 32(2): 233-240, doi: 10.1007/ s00270-008-9460-z, indexed in Pubmed: 18982387.

21. Kim SS, Kim JH, Kang HIn, et al. Ulnar nerve compression at Guyon's canal by an arteriovenous malformation. J Korean Neurosurg Soc. 2009; 45(1): 57-59, doi: 10.3340/ jkns.2009.45.1.57, indexed in Pubmed: 19242575.

22. Kusztal M, Weyde W, Letachowicz K, et al. Anatomical vascular variations and practical implications for access creation on the upper limb. J Vasc Access. 2014; 15 (Suppl 7): S70-S75, doi: 10.5301/jva.5000257, indexed in Pubmed: 24817459.

23. Layton KF, Kallmes DF, Kaufmann TJ. Use of the ulnar artery as an alternative access site for cerebral angiography. Am J Neuroradiol. 2006; 27(10): 2073-2074, indexed in Pubmed: 17110669.

24. Liu W, Lagaac R, Pettigrew GJ, et al. Outcomes after ulnar-basilic arteriovenous fistula formation. Ann Vasc Surg. 2013; 27(2): 232-237, doi: 10.1016/j.avsg.2012.04.014, indexed in Pubmed: 22981015.

25. Machiels F, Shahabpour M, De Maeseneer M, et al. Tarsal tunnel syndrome: ultrasonographic and MRI features. JBR-BTR. 1999; 82(2): 49-50, indexed in Pubmed: 10874388.

26. Mahajan R, Raheja S, Agarwal S, et al. Tortuous ulnar artery and Gantzer's muscle: a rare presentation with clinical implications. IAIM. 2015; 2(7): 141-146.

27. Moul JW, Wind GG, Wright CR. Tortuous and aberrant external iliac artery precluding radical retropubic prostatectomy for prostate cancer. Urology. 1993; 42(4): 450-452, doi: 10.1016/0090-4295(93)90384-m, indexed in Pubmed: 8212448.

28. Moullot P, Gay AM, Guidicelli T, et al. [Superficial ulnar artery while harvesting a radial forearm flap]. Ann Chir Plast Esthet. 2015; 60(1): 74-77, doi: 10.1016/j.anplas.2013.08.009, indexed in Pubmed: 24095106.

29. Nakayama Y. Introduction to fluid mechanics. Butterworth-Heinemann. 2018.

30. Natsis K, Papadopoulou A, Paraskevas G, et al. High origin of a superficial ulnar artery arising from the axillary artery: anatomy, embryology, clinical significance and a review of the literature. Folia Morphol. 2006; 65(4): 400-405, indexed in Pubmed: 17171623.

31. Oh SJ, Meyer RD. Entrapment neuropathies of the tibial (posterior tibial) nerve. Neurol Clin. 1999; 17(3): 593-615, vii, doi: 10.1016/s0733-8619(05)70154-7, indexed in Pubmed: 10393755.

32. Ortiz-Pomales $Y$, Smith J, Weiss J, et al. Tortuous axillary artery aneurysm causing median nerve compression. Ann Vasc Surg. 2014; 28(1): 122.e1-122.e3, doi: 10.1016/j. avsg.2013.07.004, indexed in Pubmed: 24189011.

33. Ostadfar A. Biofluid Mechanics: Principles and Applications. Elsevier 2016.

34. Ozgur Z, Ucerler H, Aktan Ikiz ZA. Branching patterns of the popliteal artery and its clinical importance. Surg Radiol Anat. 2009; 31(5): 357-362, doi: 10.1007/s00276-0080454-y, indexed in Pubmed: 19142562.

35. Pappu S, Dardik A, Tagare H, et al. Beyond fusiform and saccular: a novel quantitative tortuosity index may help 
classify aneurysm shape and predict aneurysm rupture potential. Ann Vasc Surg. 2008; 22(1): 88-97, doi: 10.1016/j. avsg.2007.09.004, indexed in Pubmed: 18023556.

36. Ramakrishnan G, Fontem RF, Sheth SU. Tortuous ulnar artery presenting as left distal forearm mass. J Vasc Surg Cases Innov Tech. 2020; 6(3): 430-432, doi: 10.1016/j. jvscit.2020.06.010, indexed in Pubmed: 32775848.

37. Riekkinen $\mathrm{H}$, Karkola $\mathrm{K}$, Kankainen $\mathrm{A}$. The radial artery is larger than the ulnar. Ann Thorac Surg. 2003; 75(3): 882-884, doi: 10.1016/s0003-4975(02)04557-5.

38. Rodríguez-Niedenführ M, Vázquez T, Nearn L, et al. Variations of the arterial pattern in the upper limb revisited: a morphological and statistical study, with a review of the literature. J Anat. 2001; 199(Pt 5): 547-566, doi: 10.1046/j.1469-7580.2001.19950547.x, indexed in Pubmed: 11760886 .

39. Sgueglia GA, Di Giorgio A, Gaspardone A, et al. Anatomic basis and physiological rationale of distal radial artery access for percutaneous coronary and endovascular procedures. JACC Cardiovasc Interv. 2018; 11(20): 2113-2119, doi: 10.1016/j.jcin.2018.04.045, indexed in Pubmed: 30336816.

40. Soikkonen K, Wolf J, Hietanen J, et al. Three main arteries of the face and their tortuosity. Br J Oral Maxillofac Surg. 1991; 29(6): 395-398, doi: 10.1016/0266-4356(91)90009-t, indexed in Pubmed: 1772861.

41. Sylvester PA, Stewart R, Ellis H. Tortuosity of the human splenic artery. Clin Anat. 1995; 8(3): 214-218, doi: 10.1002/ca.980080306, indexed in Pubmed: 7606595.

42. Tsutsumi K, Shimizu H. A case of a highly tortuous descending thoracic aortic aneurysm treated by surgical exclusion. SAGE Open Med Case Rep. 2020; 8: 2050313X20926440, doi: 10.1177/2050313X20926440, indexed in Pubmed: 32537162.
43. Valsecchi O, Vassileva A, Musumeci G, et al. Failure of transradial approach during coronary interventions: anatomic considerations. Catheter Cardiovasc Interv. 2006; 67(6): 870-878, doi: 10.1002/ccd.20732, indexed in Pubmed: 16649233.

44. Vincent R, Mohandas Kg R, Shivananda N, et al. Looped and tortuous ulnar artery: an erratic unilateral vascular presentation in the proximal forearm. J Clin Diagn Res. 2016; 10(6): AD03-AD04, doi: 10.7860/ JCDR/2016/20771.7948, indexed in Pubmed: 27504273.

45. Wensing PJW, Scholten FG, Buijs PC, et al. Arterior tortuosity in the femoropopliteal region during knee flexion: a magnetic resonance angiographic study. J Anat. 1995; 186: 133-139, indexed in Pubmed: 7591974.

46. Wood NB, Zhao SZ, Zambanini A, et al. Curvature and tortuosity of the superficial femoral artery: a possible risk factor for peripheral arterial disease. J Appl Physiol. 2006; 101(5): 1412-1418, doi: 10.1152/japplphysiol.00051.2006, indexed in Pubmed: 16825527.

47. Xie X, Wang Y, Zhu H, et al. Impact of coronary tortuosity on coronary blood supply: a patient-specific study. PLoS One. 2013; 8(5): e64564, doi: 10.1371/journal. pone.0064564, indexed in Pubmed: 23691249.

48. Yoo BS, Yoon J, Ko JY, et al. Anatomical consideration of the radial artery for transradial coronary procedures: arterial diameter, branching anomaly and vessel tortuosity. Int J Cardiol. 2005; 101(3): 421-427, doi: 10.1016/j. ijcard.2004.03.061, indexed in Pubmed: 15907410.

49. Zeeshan M, Ahmed F, Kanwal D, et al. Guyon's canal syndrome due to tortuous ulnar artery with DeQuervain stenosing tenosynovitis, ligamentous injuries and dorsal intercalated segmental instability syndrome, a rare presentation: a case report. Cases J. 2009; 2: 9390, doi: 10.1186/17571626-2-9390, indexed in Pubmed: 20076781. 